\title{
MICROBIAL ASPECT OF ASPIRATION PNEUMONIA IN PATIENTS WITH ALTERED CONSCIOUSNESS ADMITTED IN DHAKA MEDICAL COLLEGE HOSPITAL, BANGLADESH
}

\author{
CHOWDHURY MRA ${ }^{1}$, AZAD KAK $^{2}$, HOSSAIN MZ $^{3}$, SARDAR MH $^{4}$, SIDDIQUI MR $^{5}, \mathrm{SAAD} \mathrm{S}^{6}$, \\ RAHMAN MM ${ }^{7}$
}

\begin{abstract}
Context: Aspiration Pneumonia is a common complication in patients with altered consciousness, if general supportive care is not taken properly. There is no national study on microbial aspect of aspiration pneumonia in hospitalized patients with altered consciousness. This study was done to see the microbial aspect of aspiration pneumonia in patients with altered consciousness admitted in a tertiary level hospital in Bangladesh.

Methods: A prospective observational study was done on 50 adult patients of aspiration pneumonia with altered consciousness admitted in the medicine department of medicine, Dhaka Medical College Hospital, Dhaka, between January and December 2010. Aspiration pneumonia was confirmed by clinical examination and laboratory investigations. Hematologic measurements, chest $x$-ray, blood gas analysis, estimation of blood urea, serum creatinine and random blood sugar were done in all patients. Sputum for gram staining and culture \& sensitivity as well as blood culture \& sensitivity were done in patients who were diagnosed as aspiration pneumonia clinically and radiologically. Validated sputum was cultured in blood agar, chocolate agar and McConkey's agar media. Blood culture was done in trypticase soya broth, blood agar, chocolate agar and McConkey's agar media.

Results: The mean age was $38.9 \pm 17.3$ years. 35 (70\%) patients were male and 15 (30\%) patients were female. Blood culture was found positive in $30.8 \%$ patients who developed aspiration pneumonia among the study patients. In sputum for Gram staining, only Gram positive Cocci was present in 46.15\%, only Gram negative Bacilli was present in 38.46\%, mixed Gram positive cocci and Gram negative bacilli was present in $7.7 \%$ and Gram negative Coccobacilli was found in $7.7 \%$ patients who developed aspiration pneumonia among the study patients. In growth positive cases $(n=11)$ sputum culture revealed $45.45 \%$ streptococcus pneumoniae, 36.36\% Klebsiella pneumoniae, 9\% Pseudomonas species and 9\% Haemophylus influenzae. The mean duration of hospital stay was $8.12 \pm 2.42$ days with ranging from 4 to 14 days.
\end{abstract}

Key words: Aspiration Pneumonia, altered consciousness.

J Dhaka Med Coll. 2013; 22(2) : 110-114.

\section{Introduction:}

Aspiration pneumonia refers to the pulmonary consequences (inflammation of lung parenchyma and bronchial tubes) resulting from the abnormal entry of fluid, particulate exogenous substances, endogenous secretions or gastric contents into the lower airways. Approximately $10 \%$ of patients who are

1. Dr. Md. Rashed Alam Chowdhury, Assistant Professor, Dept. of Medicine, Jahurul Islam Medical College, Bajitpur, Kisoregonj.

2. Prof. Khan Abul Kalam Azad, Professor of Medicine, Dhaka Medical College, Dhaka.

3. Dr. Mohammad Zaid Hossain, Associate Professor of Medicine, Dhaka Medical College, Dhaka

4. Dr. Md. Hafiz Sardar, Associate Professor, Dept. of Medicine, Dhaka Medical College, Dhaka.

5. Dr. Mahmudur Rahman Siddiqui, Assistant Professor, Dept. of Medicine, Anwer Khan Modern Medical College, Dhaka.

6. Dr. Sanjeeda Saad, Post Graduate Student, Dept. of Surgery, Bangabandhu Sheikh Mujib Medical University (BSMMU), Dhaka.

7. Dr. Md. Mostafizur Rahman, Assistant Registrar, Department of Medicine, Dhaka Medical College Hospital, Dhaka Correspondence : Dr. Md. Rashed Alam Chowdhury, Assistant Professor, Dept. of Medicine, Jahurul Islam Medical College, Bajitpur, Kisoregonj. Email: rashedalamc@gmail.com 
hospitalized due to altered consciousness or drug overdose will have aspiration pneumonia. ${ }^{1}$ Aspiration pneumonia is more common in extremely young and old patients and in males than females. ${ }^{2}$ The mortality rate of aspiration pneumonia is approximately $1 \%$ in outpatient setting and upto $25 \%$ in those requiring hospitalization. The mortality rates for severe chemical pneumonitis can be up to $70 \%{ }^{3}$

Acute inflammatory phase response involves massive recruitments' of neutrophil with systemic elaboration of various cytokine (mainly IL-8) mediated cascades. However, aspiration may causes - chemical pneumonitis i.e. aspiration of gastric juice may cause fatal damage (Mendelson's Syndrome) including hemorrhagic tracheobronchitis, pulmonary edema and ARDS; bacterial infection which is typically caused by the aspiration of bacteria that normally reside in the oral cavity, upper airways or stomach especially in patients with poor oropharyngeal hygiene. The number of anaerobes is likely to be even or greater in patients with poor oral hygiene. ${ }^{4}$ True aspiration pneumonia usually refers to an infection caused by less virulent bacteria, primarily anaerobes and streptococci, which are common constituents of the normal flora in a susceptible host prone to aspiration. ${ }^{5}$ The pathogens that commonly produce aspiration pneumonia are Streptococcus pneumoniae, Haemophilus influenzae, gram-negative bacilli, Staphylococcus aureus and anaerobes (peptostreptococcus, fusobacterium nucleatum, prevortella, and bacteroides). ${ }^{6}$ Clinical features of aspiration pneumonia involving anaerobic bacteria include: Indolent symptoms, usually compromised consciousness, absence of rigor, failure to recover, sputum often has a putrid odor $^{7}$; and obstruction i.e. large volume of aspirated materials may lead to acute airway obstruction with lobar or segmental atelectasis. ${ }^{8}$

On the other hand, level of consciousness is the relative state of awareness of self and the environment and ranges from fully awake to comatose state. ${ }^{9}$ Systematic assessment of an altered conscious patient can be done by the application of the Glasgow Coma Scale (minimum score-3, maximum score-15). ${ }^{6}$
Internationally aspiration pneumonia is considered as a common disease; however, no recent statistics are available. Aspiration pneumonia in altered conscious patient is not uncommon in Bangladesh. In Bangladesh there is no study on microbiological aspect of aspiration pneumonia. This study is an attempt to identify the microbiological aspect of aspiration pneumonia in hospitalized patients with altered consciousness. There is no data currently available and the results of the present study are expected to formulate a baseline of data for future reference.

\section{Methods:}

This prospective observational study was done in the Department of Medicine of Dhaka Medical College Hospital, Dhaka, between January and December of 2010. 50 adult hospitalized patients with altered consciousness in the department of Medicine of Dhaka Medical College Hospital were enrolled in this prospective study. Informed written consent was obtained from the patient's attendant after full explanation of the disease process. Evaluation was made by history and physical examination in a structured case record form. Patients diagnosed clinically as aspiration pneumonia was screened in the study. Assessment of altered conscious patient was done by application of the Glasgow Coma Scale. Hematologic measurements, chest $\mathrm{x}-$ ray, blood gas analysis, estimation of blood urea, serum creatinine and random blood sugar were done in all patients. Sputum for gram staining and culture $\&$ sensitivity and blood culture $\&$ sensitivity were done in patients who were diagnosed as aspiration pneumonia clinically and radiologically. Validated sputum was cultured in blood agar, chocolate agar and McConkey's agar media. Blood culture was done in trypticase soya broth, blood agar, chocolate agar and McConkey's agar media. For scanty production of cough, patient's sputum was collected after nebulization by hypertonic normal saline. Other investigations such as CT scan of brain, CSF Study, LFT were done to find out the cause of altered consciousness. Categorical data was presented as frequency and percentage and continuous variable presented as mean and standard deviation. All data were processed and analyzed by SPSS version 16.0. 


\section{Results:}

This study enrolled 50 altered conscious patients. The mean age of the patients was $57.42 \pm 13.63$ years with ranged from 25 to 90 years and most of the patients $(30 \%)$ were found in the age group of $51-60$ years. 35 (70\%) patients were male and $15(30 \%)$ patients were female (Fig. 1). In sputum for gram staining, only gram positive cocci was present in $46.15 \%$, only gram negative bacilli was present in $38.46 \%$, mixed gram positive cocci and gram negative bacilli was present in $7.7 \%$ and gram negative coccobacilli was found in $7.7 \%$ patients who developed aspiration pneumonia among the study patients (Table-II). In growth positive cases (11) sputum culture revealed $45.45 \%$ streptococcus pneumoniae, $36.36 \%$ klebsiella pneumoniae, $9 \%$ pseudomonas species and 9\% haemophylus influenza (Table-III). Blood culture was found positive in $30.8 \%$ patients who developed aspiration pneumonia among the study patients (Table-IV). For streptococcus pneumoniae, more sensitive antibiotics were amoxyclav and levofloxacin (Table-V). For gram negative bacilli and coccobacilli, more sensitive antibiotics were meropenem, ceftriaxone and clarithromycin (Table-VI). The mean duration of hospital stay was $8.12 \pm 2.42$ days with ranging from 4 to 14 days.

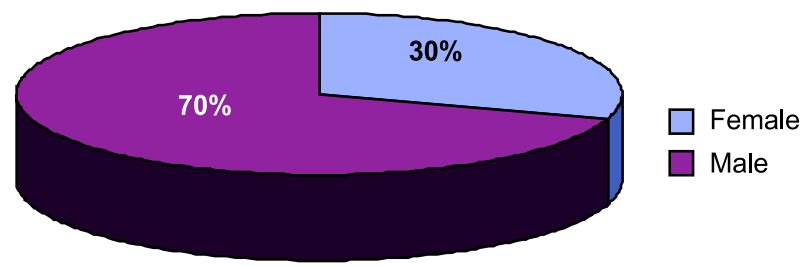

Fig.-1: Distribution of patients according to sex $(n=50)$

Table I

Age distribution of the study patients $(n=50)$

\begin{tabular}{lcc}
\hline Age in years & Number of patients & Percentage \\
\hline $25-40$ & 06 & 12 \\
$41-50$ & 10 & 20 \\
$51-60$ & 15 & 30 \\
$61-70$ & 13 & 26 \\
$71-80$ & 04 & 08 \\
$81-90$ & 02 & 04 \\
\hline
\end{tabular}

Mean \pm SD $=57.42 \pm 13.63$

Range $(\min -\max )=(25-90)$
Table-II

Distribution of the study patients according to sputum for gram staining $(n=13)$

\begin{tabular}{lcc}
\hline $\begin{array}{l}\text { Sputum for Gram } \\
\text { staining }\end{array}$ & $\begin{array}{c}\text { Number of } \\
\text { patients }\end{array}$ & Percentage \\
\hline Only Gram positive cocci & 06 & 46.15 \\
Only Gram negative bacilli & 05 & 38.46 \\
Mixed Gram positive cocci & 01 & 7.7 \\
and Gram negative bacilli & & \\
Gram negative coccobacilli & 01 & 7.7 \\
\hline
\end{tabular}

Table-III

Result of sputum culture positive cases of patients with aspiration pneumonia $(n=11)$

\begin{tabular}{lcc}
\hline Sputum Culture & $\begin{array}{c}\text { Number of } \\
\text { patients }\end{array}$ & Percentage \\
\hline Streptococcus pneumoniae & 05 & 45.45 \\
Klebsiella pneumonia & 04 & 36.36 \\
Pseudomonas species & 01 & 09.00 \\
Haemophylus influenzae & 01 & 09.00 \\
\hline
\end{tabular}

Table-IV

Blood culture of the study patients ( $n=13)$

\begin{tabular}{lcc}
\hline Blood culture & $\begin{array}{c}\text { Number of } \\
\text { patients }\end{array}$ & \\
\hline Streptococcus pneumoniae & 02 & 15.4 \\
Klebsiella pneumonia & 01 & 07.7 \\
Pseudomonas species & 01 & 07.7 \\
\hline
\end{tabular}

Table-V

Antimicrobial susceptibility pattern against Streptococcus pneumoniae $(n=5)$

\begin{tabular}{lccc}
\hline $\begin{array}{l}\text { Sensitive } \\
\text { Antibiotics }\end{array}$ & $\begin{array}{c}\text { Number of } \\
\text { patients (n) }\end{array}$ & Sensitive & Percentage \\
\hline Amoxyclav & 5 & 4 & 80 \\
Levofloxacin & 5 & 3 & 60 \\
Azithromycin & 5 & 3 & 60 \\
Cefixime & 5 & 1 & 20 \\
Doxycycline & 5 & 1 & 20 \\
\hline
\end{tabular}


Table-VI

Antimicrobial susceptibility patterns of isolated organisms in the study patients $(n=6)$

\begin{tabular}{|c|c|c|c|c|c|c|}
\hline \multirow[t]{2}{*}{$\begin{array}{l}\text { Sensitive } \\
\text { Antibiotics }\end{array}$} & \multicolumn{2}{|c|}{$\begin{array}{c}\text { Klebsiella } \\
\text { pneumoniae } \\
(\mathrm{n}=4)\end{array}$} & \multicolumn{2}{|c|}{$\begin{array}{l}\text { Pseudomonas } \\
\text { species } \\
(\mathrm{n}=1)\end{array}$} & \multicolumn{2}{|c|}{$\begin{array}{l}\text { Haemophylus } \\
\text { influenzae } \\
(\mathrm{n}=1)\end{array}$} \\
\hline & $\mathrm{N}$ & $\%$ & $\mathrm{~N}$ & $\%$ & $\mathrm{~N}$ & $\%$ \\
\hline Meropenem & 4 & 100 & 1 & 100 & 1 & 100 \\
\hline Ceftriaxone & 3 & 75 & 1 & 100 & 1 & 100 \\
\hline Clarithromycin & 3 & 75 & 0 & 0.0 & 1 & 100 \\
\hline Ciprofloxacin & 2 & 50 & 0 & 0.0 & 0 & 0.0 \\
\hline Amoxyclav & 1 & 25 & 0 & 0.0 & 0 & 0.0 \\
\hline Cefixime & 1 & 25 & 0 & 0.0 & 1 & 100 \\
\hline
\end{tabular}

\section{Discussion:}

In this small series of patients with altered consciousness in a tertiary level hospital, the mean $( \pm \mathrm{SD})$ age was $57.42 \pm 13.63$ years with ranged from 25 to 90 years. El-Solh et al. ${ }^{1}$ found that the mean age was $80.2 \pm 6.5$ years in their study. In this study, out of 50 patients, 35 of the patients were male and 15 were female. El-Solh et al. ${ }^{1}$ found that out of 69 patients, 43 were males and 26 were females. In present study, all the patients had altered consciousness. GCS was ranging from 7 to 12 in all study patients. Streptococcus pneumoniae and klebsiella pneumoniae were the predominant organisms found in about $80 \%$ of the patients who developed aspiration pneumonia among the study patients. Blood culture was positive in $4(30.8 \%)$ patients. Streptococcus pneumoniae was found in two (15.4\%) patients who developed aspiration pneumonia and Klebsiella pneumonia was found in one $(7.7 \%)$ patient. Pseudomonas species was found in one patient. Bartlett and Gorbach $^{4}$ found anaerobic bacteria in 50 patients $(93 \%)$ and were the only pathogens in 25 patients $(46 \%)$ in their study. The predominant organisms were bacteroides and fusobacteria. Aerobic and facultative bacteria were present in 29 patients (54 per cent), but anaerobes were present concurrently in all. Lorber and Swenson ${ }^{10}$ found anaerobic bacteria in 21 of 24 community-acquired infections and in 13 cases anaerobes were the only isolates. In the hospital-acquired infections, anaerobes were found in 8 of 23 cases. Gram-negative facultative anaerobes and aerobes were common in the hospital-acquired infections. Community and hospital acquired aspiration pneumonias are bacteriologically different. The causative organisms reflect the oropharyngeal flora and the anaerobic bacteria seem to be important pathogens in both community and hospital acquired cases. El-Solh et al. ${ }^{1}$ found gram-negative enteric bacilli in $49 \%$ patients, followed by anaerobic bacteria in $16 \%$ patients, staphylococcus aureus in $12 \%$ patients and polymicrobial in $22 \%$ patients.

\section{Conclusion:}

The present study showed that $26 \%$ of hospitalized patients with altered consciousness developed aspiration pneumonia. Streptococcus and klebsiella were common organisms as identified by sputum culture. It represents the aerobic microorganisms that are likely to colonize dental plaques or oropharyngeal cavity at the time of aspiration. For Aspiration pneumonia associated with streptococcus pneumoniae sensitivity results were in favor of amoxyclav and levofloxacin. For aspiration pneumonia associated with gram negative bacilli sensitivity status was in favor of meropenem, ceftriaxone and clarithromycin respectively.

Only 50 samples were taken due to study time limit, and, therefore, the sample size is relatively small and area coverage planned is relatively small compared to total aspiration pneumonia prevalent area in the country. The sputum culture negative patient could not be 
evaluated further due to unavailability of tests (viral isolation) in the set up. Anaerobic culture of sputum and blood could not be done due to unavailability of tests in the set up. However, it requires further studies from different levels of hospitals. A nationally representative surveillance system for aspiration pneumonia may replace periodic small studies.

\section{References:}

1. El-Solh AA, Pietrantoni C, Bhat A, Aquilina AT, Okada M, Grover V, et al. Microbiology of severe aspiration pneumonia in institutionalized elderly. Am J Respir Crit Care Med 2003; 167(12): 16504.

2. Marik PE. Aspiration pneumonitis and aspiration pneumonia. N Engl J Med 2001; 344(9): 665-71.

3. DeLegge MH. Aspiration pneumonia: incidence, mortality, and at-risk populations. J Parenter Enteral Nutr 2002; 26(6 Suppl): 19-25.
4. Bartlett JG, Gorbach SL. The triple threat of aspiration pneumonia. Chest 1975; 68: 560.

5. Finegold SM, George WL, Mulligan ME. Anaerobic infections. Dis Mon 1985; 31: 1.

6. Adnet F, Baud F. Relation between Glasgow Coma Scale and aspiration pneumonia. Lancet 1996; 348(9020): 123-4.

7. Bartlett JG. Anaerobic bacterial pneumonitis. Am Rev Respir Dis 1979; 119(1): 19.

8. Fauci AS, Braunwald E, Kasper DL, Hauser SL, Longo DL, Jameson JL, et al, editors. Harrison's Principles of Internal Medicine: $.17^{\text {th }}$ ed. 2008. p. 2486.

9. Allen CMC, Lueck CJ, Dennis M. Neurological disease. In: Colledge NR, Walker BR, Ralston SH, eds. Davidson's principles and practice of medicine. $21^{\text {st }}$ ed. Edinburgh: Churchill Livingstone; 2010. p.1131-235.

10. Lorber B, Swenson RM. Bacteriology of aspiration pneumonia: a prospective study of community and hospital-acquired cases. Ann Intern Med 1974; 81: 329. 